\title{
An Acoustic Analysis of the Separation of Pitch and Stress in Onondaga $^{*}$
}

\author{
HEIKE LEHNERT-LEHOUILLIER
}

University at Buffalo (SUNY)

\section{Introduction}

Stress in many Northern Iroquoian languages is said to be phonetically realized by higher F0 and greater amplitude on the stressed syllable (Chafe 1977, Woodbury 1992 for Onondaga). For Onondaga, however, it has been claimed that pitch and stress have systematically separated under certain circumstances (Chafe 1977; Michelson 1988; Woodbury 1992, 2003).

Onondaga has retained Proto-Northern Iroquoian penultimate word accent. The separation of stress and pitch has been described as occurring mainly in words of four or more syllables such that the stressed penultimate syllable is realized with greater amplitude and the highest pitch moves leftwards (Woodbury 1992). However, Woodbury also notes that the "rules governing the choice of the syllable to receive high pitch when it separates from heavy stress are statistical" (Woodbury 1992:743). While there is a strong tendency for the pretonic vowel to receive the highest pitch, as shown in (1), the landing site of the highest pitch may also be left of the pretonic syllable, as seen in (2) (underline indicates highest pitch and an acute accent indicates stress, examples from Woodbury 1992:743744).

(1) /teyũtata?wẽthwáska?/

'they used to kill each other'

(2) /tũsahatihsihá:kwa?/

'they are unplugging it'

This variability in pitch placement is twofold: there is the just-described variability in the location of the highest $\mathrm{F} 0$, but there is also variability across as well as within speakers as to where the highest F0 will be realized (Woodbury 1992). These facts raise the question of whether this change in the word-prosodic

* I am grateful to my consultant Nora Carrier, to Hanni Woodbury for providing me with additional recordings, and to Karin Michelson for helpful comments. This work was supported by a grant from the Phillips Fund. 


\section{Heike Lehnert-LeHouillier}

system of Onondaga, which is assumed to be systematic, is in fact a phonetic phenomenon known as early peak where the F0 peak associated with a certain syllable is realized prior to this syllable. The current paper investigates this possibility by analyzing the fundamental frequency, F0 - the acoustic correlate of pitch - in the speech of two native speakers of Onondaga. For the purpose of this paper, it is assumed that the phonetic correlate of stress in Onondaga is greater amplitude over the stressed syllable. However, due to the way the recordings were obtained, the phonetic correlate of stress could not be investigated in the current acoustic analysis and the exact phonetic correlates of stress in Onondaga still await instrumental investigation.

First, the circumstances under which a separation of stress and pitch have been reported to occur in Onondaga will be summarized. Next, the research question and methodology are outlined. Then a presentation of the results follows, and finally, the findings will be discussed in light of whether this separation of pitch and stress should be regarded as a phonological or phonetic property of Onondaga.

\section{Separation of Stress and Pitch in Onondaga}

The word-prosodic system of Onondaga distinguishes between utterance-medial and utterance-final words. The separation of pitch and stress has only been reported to occur in utterance-final forms. Hence, this investigation and the following description are limited to utterance-final forms only.

As mentioned above, Onondaga has penultimate word accent with the exception of some words containing certain morphemes, some of which attract stress to the antepenultimate syllable and others to the final syllable. The penultimate accent is manifested in the phonology by tonic lengthening of vowels in an open syllable. Phonetically it is said to be realized with greater amplitude and with higher pitch in words of less than four syllables. While a separation of highest pitch and stress in words with penultimate stress is said to occur only in words of four or more syllables, words with final stress always exhibit a separation of stress and pitch. There is said to be a strong tendency for the highest pitch to be realized on the pretonic syllable in those cases where pitch separates from stress, however with two notable exceptions: a) In words with penultimate stress of four or more syllables containing an epenthetic [a], called the "joiner vowel," this [a] often repels the highest pitch such that it tends to be realized on the syllable to the left of the "joiner." b) In words with final stress of four or more syllables, the highest pitch is only realized on the pretonic syllable $=$ penultimate) if it contains a long vowel; otherwise it is realized on the antepenultimate. ${ }^{1}$ The pattern of the separation of pitch and stress for words with penultimate and final stress is summarized in the tables in (3) and (4) below. For

${ }^{1}$ This pattern is stated in Michelson (1988) but not explicitly in either Woodbury (1992) or (2003). 
words with antepenultimate stress, the highest pitch is said to always occur in the pretonic syllable.

(3) Separation of Pitch and Stress in Words with Penultimate Accent

\begin{tabular}{|l|l|l|}
\hline & Stress & F0 Peak \\
\hline a) Words of $3 \sigma$ & Penultimate & Penultimate \\
\hline b) Words of 4 or more $\sigma$ & Penultimate & Antepenultimate or earlier \\
\hline Words with "joiner" [a] as antepenult & Penultimate & Third to last or earlier \\
\hline
\end{tabular}

(4) Separation of Pitch and Stress in Words with Final Accent

\begin{tabular}{|l|l|l|}
\hline & Stress & F0 Peak \\
\hline a) Words of $3 \sigma$ & Final & Penultimate or earlier \\
\hline b) Words of 4 or more $\sigma$ with V: & Final & Penultimate or earlier \\
\hline c) All other words of 4 or more $\sigma$ & Final & Antepenultimate or earlier \\
\hline
\end{tabular}

\section{Research Question and Methodology}

\subsection{Research Question}

The research question is set within an Autosegmental-Metrical framework that assumes the distinction between alignment and association of F0 events (Ladd 1983, 1996). Alignment is understood as the phonetic property of the relative timing of the F0 event with respect to the segmental string, whereas association refers to the phonological property that defines to which abstract domain, i.e. syllable, the F0 event belongs. Given this distinction, an F0 event may be aligned early, late, or even outside the syllable with which it is associated.

The research question is twofold:

A) Does the acoustic analysis confirm the above-described pattern of separation of pitch and stress that has been postulated based on impressionistic data?

B) Is the separation of pitch and stress due to an early peak alignment pattern in which the F0 peak occurs early in or even before the stressed syllable?

\subsection{Data}

Recordings from 2 native speakers, one female and one male, were analyzed for this study. The recordings were digitized at 16 bits with a sampling rate of 22050 $\mathrm{Hz}$ and were analyzed using PRAAT (Boersma and Weenink 2005).

The data was grouped into three data sets: Data set 1 consists of 100 words read in isolation by the female speaker; data set 2 consists of 13 utterance-final words taken from a short narrative by the same speaker; and data set 3 consists of 50 utterance-final words taken from a long (30 minutes) narrative by the male speaker. Due to the infrequency of words with antepenultimate stress in the narrative, only words with penultimate stress and final stress were investigated.

The internal make-up of the three data sets is summarized in the table in (5). Due to the very limited number of tokens in data set 2 as well as its imbalanced 


\section{Heike Lehnert-LeHouillier}

distribution, any results obtained from this data set have to be regarded with suspicion and can only be taken as possible trends in the narrative data which have to be confirmed by the longer narrative in data set 3 .

The three sets together yield a total of 162 words containing 717 vowels. All vowels were labeled in order to determine the highest F0 over each vowel as well as the F0 at the midpoint of the vowel. Furthermore, the overall duration of each vowel was measured and the point at which the F0 peak occurred was determined.

$$
\text { Distribution of Words by Number of Syllable in the Data Sets }
$$

\begin{tabular}{|c|c|c|c|}
\hline & \multicolumn{3}{|c|}{ Number of words in each data set } \\
\hline Number of $\boldsymbol{\sigma}$ per word & Set $\mathbf{1}$ & Set 2 & Set 3 \\
\hline 3 syllables & 20 & 2 & 10 \\
\hline 4 syllables & 20 & 3 & 10 \\
\hline 5 syllables & 20 & 3 & 10 \\
\hline 6 syllables & 20 & 2 & 10 \\
\hline Final stress & 20 & 2 & 10 \\
\hline TOTAL & $\mathbf{1 0 0}$ & $\mathbf{1 2}$ & $\mathbf{5 0}$ \\
\hline
\end{tabular}

\subsection{Methodology}

In order to answer the first part of the research question, which is to confirm or disconfirm the reported pattern of separation of pitch and stress as summarized in section 1, the syllable with the highest pitch was determined for each word and it was evaluated whether or not it coincided with the stressed syllable. The stressed syllable was taken to be the syllable which is the most prominent syllable in a word according to previous descriptions.

The second part of the research question - as declared above - aims at investigating whether the separation of pitch and stress could be due to an early peak pattern prevalent in the language. In order to determine whether an early peak pattern is present in the language, two different evaluation procedures were applied according to whether the highest F0 peak was realized on the stressed syllable or whether it occurred prior to the stressed syllable. For all words with no separation of F0 and stress, it was determined whether the highest F0 peak occurred early in the stressed syllable. 'Early' was defined as within the first $1 / 4$ of the stressed syllable. For all words with the highest F0 peak prior to the stressed syllable, it was determined whether this F0 peak was the only F0 peak that could be associated with the stressed syllable by evaluating whether or not another albeit less prominent - F0 peak was present on the stressed syllable. Only in those cases in which the highest F0 peak in a word did not co-occur with another less prominent F0 peak on the stressed syllable, was it concluded that an early peak was present. An example of an early peak - in this case the highest F0 occurs on the antepenultimate syllable - is given in the figure in (6). 
(6) Example of Early Peak with Highest F0 on Antepenultimate Syllable /ota?kæ: té: njũ?/ 'snow is falling lightly'

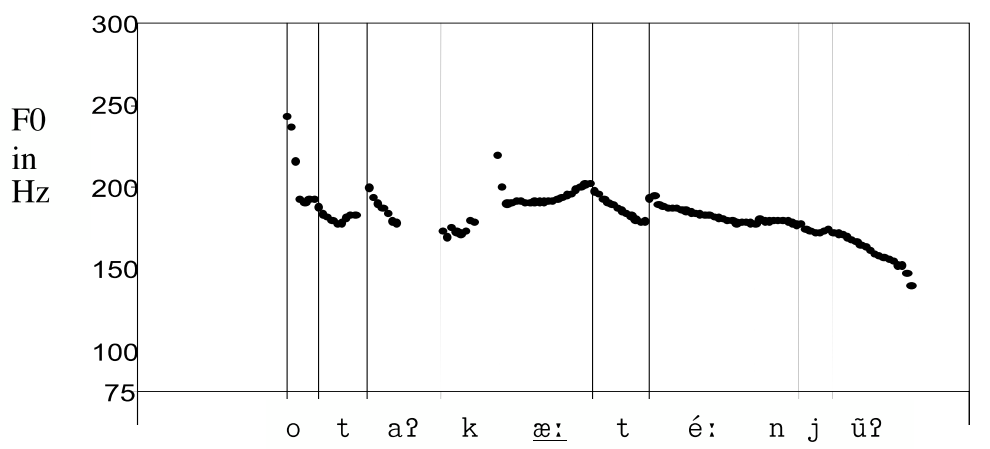

The figure in (7) shows an example in which the highest F0 peak also occurs on the antepenultimate syllable, however in this case the penultimate $(=$ stressed syllable) also has its own, less prominent F0 peak.

(7) Example of Highest F0 on Antepenultimate Syllable but no Early Peak /onihnotákwa?/ 'apron'

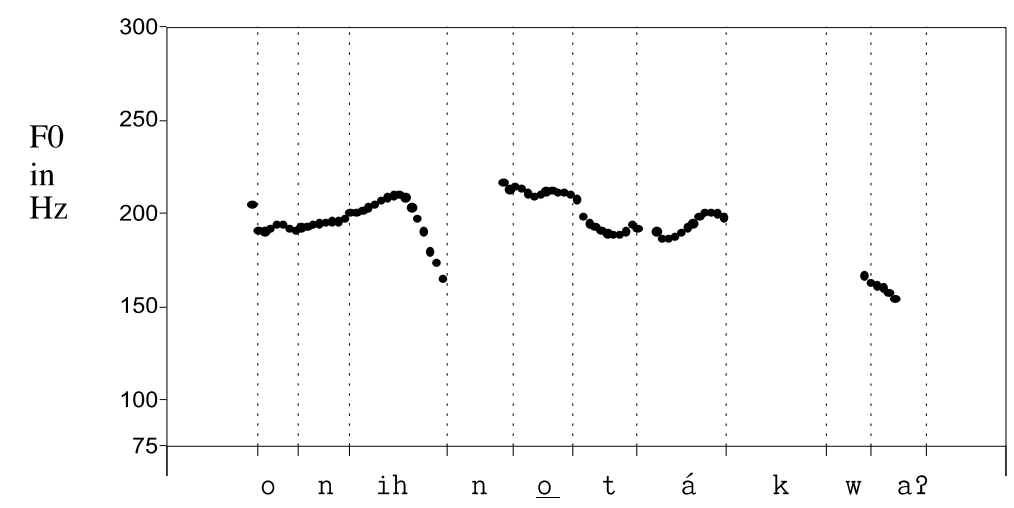

\section{Results}

3.1. Pattern of Separation of Fo from Stress

First, the question of how frequently the highest F0 peak does not coincide with the stressed syllable is addressed. Out of the total of 162 words $45 \%(73 / 162)$ show a separation of highest F0 from the stressed syllable. However, only 130 of the words - those with four or more syllables and those with final stress - are expected to exhibit a separation of pitch and stress and 55\% (72/130) of these do 


\section{Heike Lehnert-LeHouillier}

indeed show a separation of F0 and stress. While only one of the three-syllable words showed the highest F0 peak on a different syllable than the stressed syllable, the percentage of words where the highest F0 peak and the stressed syllable do not coincide increases with the number of syllables in a word. The detailed results are given in the table in (8).

Percentage of words where F0 and stress did NOT coincide

\begin{tabular}{|c|cc|cc|ccc|c|}
\hline & \multicolumn{2}{|c|}{ Set 1 } & \multicolumn{2}{|c|}{ Set 2 } & \multicolumn{2}{c|}{ Set 3 } & \multicolumn{2}{c|}{ Total } \\
\hline 3 syllabic & $5 \%$ & $(1 / 20)$ & $0 \%$ & $(0 / 2)$ & $0 \%$ & $(0 / 10)$ & $3 \%$ & $(1 / 32)$ \\
\hline 4 syllabic & $45 \%$ & $(9 / 20)$ & $33 \%$ & $(1 / 3)$ & $30 \%$ & $(3 / 10)$ & $39 \%$ & $(13 / 33)$ \\
\hline 5 syllabic & $50 \%$ & $(10 / 20)$ & $66 \%$ & $(2 / 3)$ & $40 \%$ & $(4 / 10)$ & $48 \%$ & $(16 / 33)$ \\
\hline 6 syllabic & $70 \%$ & $(14 / 20)$ & $50 \%$ & $(1 / 2)$ & $30 \%$ & $(3 / 10)$ & $56 \%$ & $(18 / 32)$ \\
\hline Final stress & $95 \%$ & $(19 / 20)$ & $0 \%$ & $(0 / 2)$ & $60 \%$ & $(6 / 10)$ & $78 \%$ & $(25 / 32)$ \\
\hline
\end{tabular}

Thus far, it was determined whether or not the syllable with the highest F0 value and the stressed syllable coincide. Next, the location of the syllable with the highest F0 peak within a word is shown. The graph in (9) shows the location of the syllable with the highest F0 with respect to the stressed syllable. S, indicated by a checked pattern, refers to the stressed syllable (i.e. the penultimate syllable, or the final syllable in those words with final stress), S-1 refers to the syllable to the left of the stressed syllable (= pretonic syllable; indicated by black dots), S-2 refers to the syllable to the left of the pretonic (diagonal stripes), and so on. S-3 is indicated with white dots on black background, and S-4 with a grid pattern. The cases labeled as 'other' are instances in which it was not possible to determine which syllable had the highest F0 because more than one syllable had equally high values $(+/-4 \mathrm{~Hz})$. The graph in (9) shows the percentages for all three data sets combined since the distribution was fairly similar across the three sets.

(9) Location of Highest F0 by Syllable

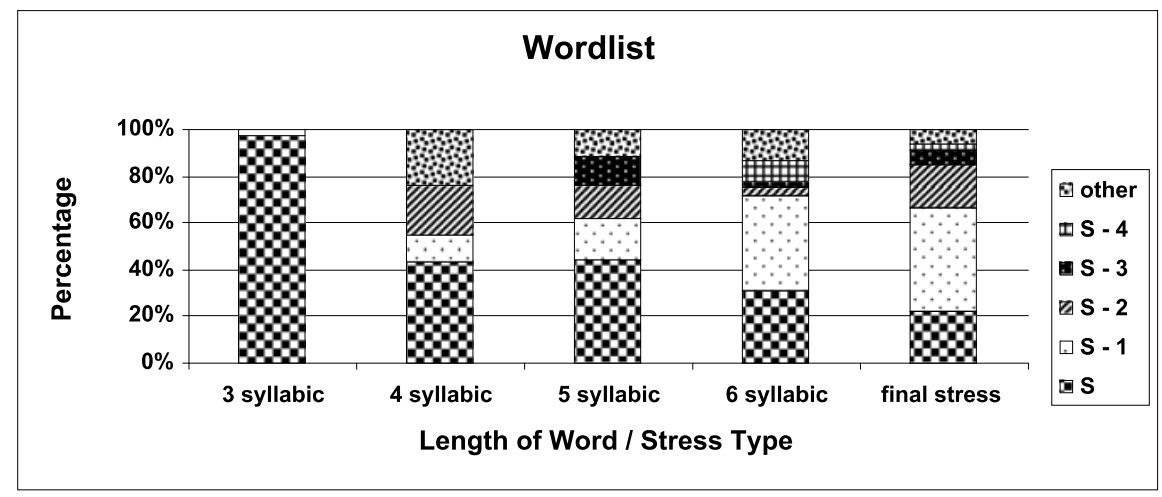




\section{Separation of Pitch and Stress in Onondaga}

Does the acoustic analysis support the pattern of separation of pitch and stress as reported in Woodbury $(1992,2003)$ and Michelson (1988)? As for words with penultimate accent, the claim that words of three syllables do not show a separation of pitch and stress is clearly supported by the acoustic record (97\% show no separation). It is also the case that in many words (55\%) of four or more syllables the syllable with the highest F0 peak is different from the stressed syllable (see the table in (8)). However, in words with final accent we do find contrary to what was reported - that the highest F0 does sometimes (22\% of the cases) fall on the final syllable. Furthermore, the pattern reported in Michelson (1988) that the default location of the highest pitch in words with final stress is the antepenultimate syllable and that the penultimate syllable receives the highest F0 only if its vowel is long is also not supported by the acoustic data. In fact, with $44 \%$ percent of all words with final stress, the penultimate syllable is the most common location of the highest $\mathrm{F} 0$ in these words regardless of whether its vowel is long or not. In words with penultimate stress, the reported strong tendency for the highest F0 to occur on the antepenultimate syllable is only apparent in words of six syllables (41\%). In four-syllable words only $12 \%$ showed the highest F0 on the antepenult, and in words of five syllables $18 \%$ had the highest F0 on the pretonic syllable. The syllable to the left of the pretonic also carried the highest F0 peak, but to a lesser extent: in five-syllable words $15 \%$, in words with final stress $19 \%$, and in four-syllable words - where this syllable constitutes the initial syllable of the word - the highest F0 peak was on this syllable in $21 \%$ of all cases. The highest F0 peak also occurred on the initial syllable of five-syllable words $(12 \%)$ and of six-syllable words (9\%). The occurrence of the highest F0 peak on any other syllable was less frequent than $5 \%$. The percentage values are summarized in the table in (10). Note that only for words with final stress, the stressed syllable is the final syllable; for all others, the stressed syllable is the penultimate syllable.

(10) Percentage Values of Location of Highest F0 by Syllable

\begin{tabular}{|l|l|l|l|l|l|l|}
\hline & Stressed = S & S - 1 & S - 2 & S - 3 & S - 4 & Other \\
\hline 3 syllabic & $97 \%$ & $3 \%$ & & & & \\
\hline 4 syllabic & $43 \%$ & $12 \%$ & $21 \%$ & & & $24 \%$ \\
\hline 5 syllabic & $45 \%$ & $18 \%$ & $15 \%$ & $12 \%$ & & $12 \%$ \\
\hline 6 syllabic & $31 \%$ & $41 \%$ & $3 \%$ & $3 \%$ & $9 \%$ & $13 \%$ \\
\hline Final stress & $22 \%$ & $44 \%$ & $19 \%$ & $6 \%$ & $3 \%$ & $6 \%$ \\
\hline
\end{tabular}

\subsection{Early Peak Pattern}

While the previous section established with which of the syllables in a word the highest F0 peak was aligned, this section aims to determine whether an early peak pattern is prevalent in Onondaga. As discussed in section 2.3, an early peak is assumed to be present if either of these conditions holds: 


\section{Heike Lehnert-LeHouillier}

a) The F0 peak on a stressed syllable occurs early (i.e. within the first $1 / 4$ of the syllable).

b) The F0 peak aligned with a syllable prior to the stressed syllable can be associated with the stressed syllable (i.e. if the stressed syllable has no F0 peak on its own).

The graphs in (11), (12), and (13) show the percentage of occurrences of early peaks in each of the three data sets. The graph in (11) presents the results from data set 1 (wordlist), (12) shows the results from data set 2 (short narrative), and (13) shows how often an early peak occurred in data set 3 (long narrative). The percentage of early peaks was calculated over the number of words that could possibly exhibit an early peak; all cases listed under 'Other' in the graph in (9) and in the table in (10) were not included.

(11) Percentage of Occurrence of Early Peaks in Data Set 1

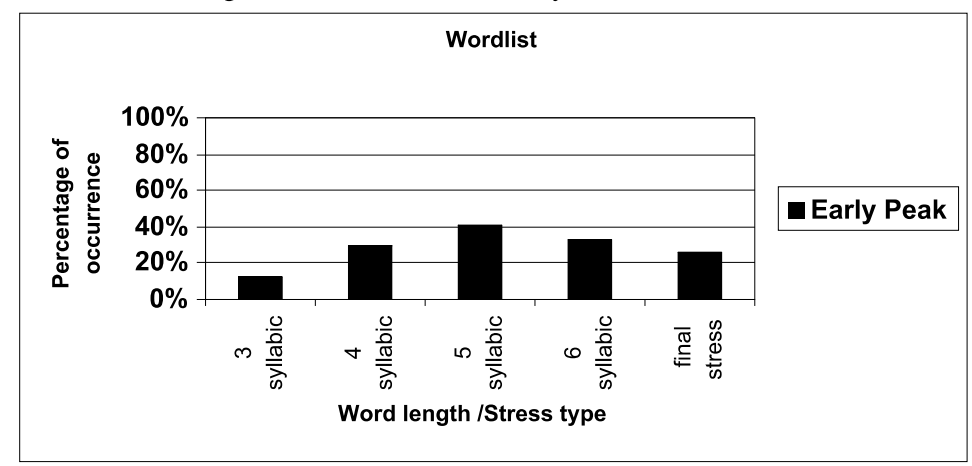

(12) Percentage of Occurrence of Early Peaks in Data Set 2

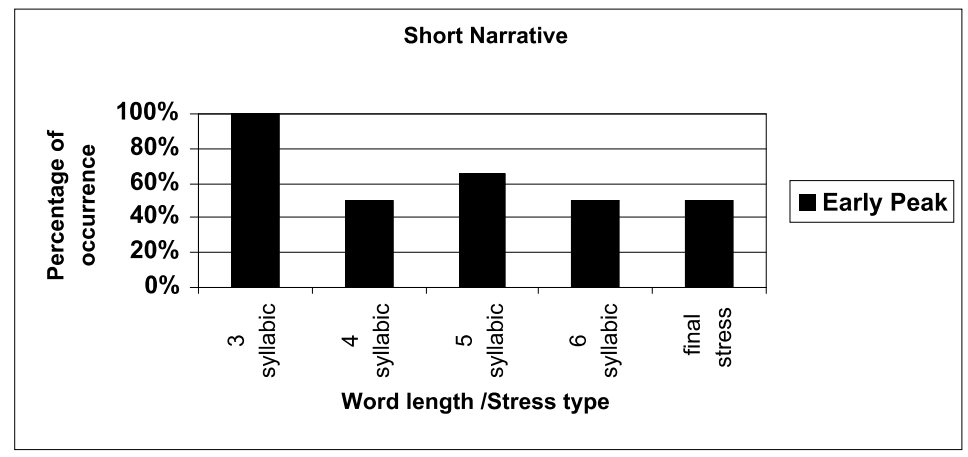




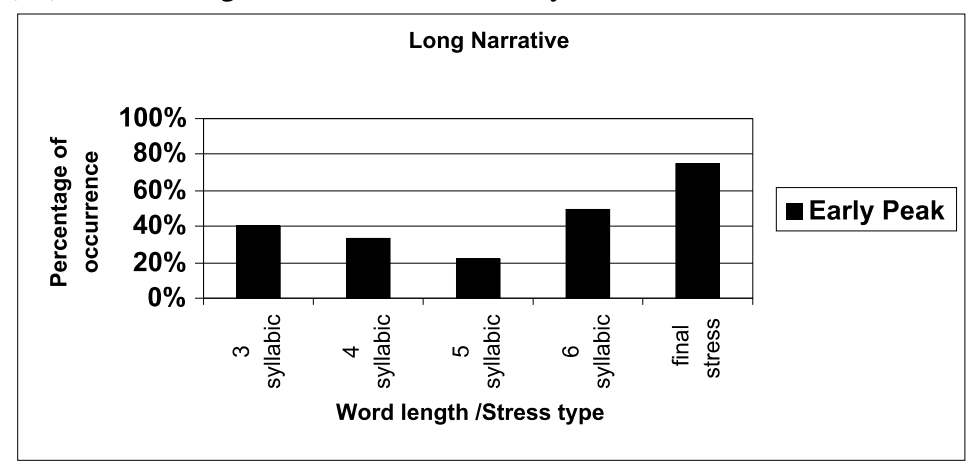

Overall, $36 \%$ of all words exhibit an early peak, and $30 \%$ of those cases where stress and pitch are separated show an early peak. Turning to the different data sets, we notice a difference in the frequency of occurrence of early peaks in the wordlist data compared to the narrative data, with the narratives showing a higher percentage of early peaks. The overall percentage of early peaks in data set 1 , the wordlist, is $32 \%$, while the percentage of early peaks in data set 2 , the short narrative by the same speaker, is twice as high: $64 \%$. Due to the small number and the imbalanced distribution of words in data set 2 , we need to look at data set 3 , the longer narrative, to confirm this tendency. The results from data set 3 do confirm the trend and show that the number of early peaks is also higher in this narrative data even though at $43 \%$ it is not as high as in data set 2 . A closer look at the detailed results presented in the graphs on the previous page reveals that the biggest difference between the wordlist items and the narrative data lies in the number of early peaks in six-syllable words and words with final stress. The narrative data show much higher percentages of early peaks in the 6 syllabic words (50\% each) and the words with final stress (50\% set 2 and $75 \%$ set 3 ). This in connection with the finding from section 3.1, especially the results presented in (8), which showed that six-syllable words and words with final stress exhibit the highest percentage of separation of stress and pitch, might indicate that the separation of pitch and stress in Onondaga has its roots in discourse prosody and was possibly generalized to the domain of word prosody. While this result seems to support the early peak hypothesis stating that the separation of stress and pitch might have arisen out of an early peak pattern, it leaves one question unanswered.

All cases in which an early peak occurred in a syllable prior to the stressed syllable are cases where the highest F0 occurred on the pretonic syllable, never on a syllable left of the pretonic. While it is possible for an early peak to occur more than one syllable early, the fact that no such case was found in this data calls into question the early peak hypothesis due to the existence of words with the highest F0 peak as early as 4 syllables to the left of the stressed syllable, as shown in (9) 


\section{Heike Lehnert-LeHouillier}

and (10) above. This leads to the question of whether alternative approaches fare better at explaining the separation of pitch and stress in Onondaga.

\section{Discussion and Conclusion}

The main hypothesis investigated in this paper was whether the separation of pitch and stress in Onondaga, which has been proposed to be a phonological phenomenon (Chafe 1977; Michelson 1988; Woodbury 1992, 2003), is not in fact of a lower-level phonetic nature. Assuming the distinction between phonetic alignment and phonological association, the acoustic record of a total of 162 words has been analyzed and searched for alignment patterns that show an early alignment of the highest F0 peak of a word early in or prior to the stressed syllable but such that it might still be associated with the stressed syllable. The results have shown that early peaks can account for only $30 \%(22 / 73)$ of all cases in the three data sets in which the highest F0 peak did not coincide with the stressed syllable. While one might argue based on the results presented above that the separation of stress and pitch might have arisen out of early peak pattern found in discourse prosody and subsequently become generalized into the wordprosodic domain, this leaves unaccounted why this generalization process has left behind a rather large residue (55\%). Furthermore, a phonetic account of the separation of pitch and stress in terms of an early peak pattern leaves unanswered the question of why the highest F0 peak can get realized on syllables as far as 4 syllables before the stressed syllable.

An alternative approach to the separation of pitch and stress - one that seeks a phonological rather than a phonetic explanation - is hinted at in Prince (1983) and Hayes (1995). They assume that the Northern Iroquoian accentual system is partly metrical and partly tonal. Hayes furthermore notes that the Onondaga accentual system - similar to that of Cayuga and Seneca - has changed from a penultimate system to an iambic left-to-right system as evidenced in second syllable lengthening. The phonological hypothesis, then, would be that the high tone that in Proto Northern Iroquoian was located on the strongest syllable (in most cases the penultimate) could now have taken over a new functional load in Onondaga and instantiate the newer left-to-right iambic system. If this was the case, we should expect the highest F0 peak in a word to occur only on even numbered syllables. This, then, would explain why no separation of pitch and stress occurs in three syllable words since the stressed syllable always coincides with the second syllable. However, it would not explain why the separation of pitch and stress is rather frequent in words with final stress. A closer look at the different length words with penultimate stress shows that we do not - as expected under this hypothesis - find a more frequent occurrence of F0 peaks on even-numbered syllables. The table in (14) shows again the percentage of occurrence of the highest F0 peak by syllable - as the table in (10) did - but this time the results are displayed in absolute order of syllables rather than relative to the stressed syllable. The even-numbered syllables are highlighted. All cases with no clear F0 peak on a single syllable are omitted, hence the values in each row do not add up to $100 \%$. 
Separation of Pitch and Stress in Onondaga

(14) Percentage Values of Location of Highest F0 by Syllable

\begin{tabular}{|l|l|l|l|l|l|l|}
\hline & $1^{\text {st }}$ & $2^{\text {nd }}$ & $3^{\text {rd }}$ & $4^{\text {th }}$ & $5^{\text {th }}$ & $6^{\text {th }}$ \\
\hline 3 syllabic & $3 \%$ & $97 \%$ & $0 \%$ & & & \\
\hline 4 syllabic & $21 \%$ & $12 \%$ & $43 \%$ & $0 \%$ & & \\
\hline 5 syllabic & $12 \%$ & $15 \%$ & $18 \%$ & $45 \%$ & $0 \%$ & \\
\hline 6 syllabic & $9 \%$ & $3 \%$ & $3 \%$ & $41 \%$ & $31 \%$ & $0 \%$ \\
\hline
\end{tabular}

The table in (14) makes apparent that the only case in which the even-numbered antepenultimate wins out over an odd-numbered penultimate syllable is in words of six syllables. The separation of pitch and stress in six-syllable words, however, can also be accounted for by invoking the phonetically motivated early peak hypothesis, as seen in section 3. Crucially, if the highest F0 peak in a word were to instantiate a left-to-right iambic system, we should not find the pattern seen for four-syllable words in which the two odd-numbered syllables $\left(1^{\text {st }}\right.$ and $\left.3^{\text {rd }}\right)$ are much more often the location of the highest F0 than the even-numbered second syllable.

In conclusion, it can be said that the separation of stress and pitch in Onondaga is neither completely phonetically motivated nor driven by the instantiation of a new metrical system. While certain aspects such as the increase of separation of stress and pitch with increasing word length, the existence of an early peak pattern in at least a subset of cases, and the reported within-speaker variability strongly point towards a phonetic rather than a phonological explanation, the phonetic characteristics do not explain everything. Future research has to clarify what exactly the tonal properties of Onondaga are. While previous descriptions seem to suggest that there is always only one high tone per word (especially Chafe 1977), 11\% of words in the current data showed an F0 value of equal height on at least two consecutive syllables. Furthermore, the lexical character of the separation of stress and pitch needs more investigation. The current study focused on the number of syllables in a word and whenever more than one token of a word was collected, only one was included for analysis. However, it was noted that despite Woodbury's (1992) report of within-speaker variability, all tokens of the same word showed the same pattern of separation of stress and pitch. Finally, a perceptual study that would test to what extent speakers of Onondaga are sensitive to the tonal characteristics of their language would greatly aid the clarification of the importance of the separation of pitch and stress in this language. 


\section{Heike Lehnert-LeHouillier}

\section{References}

Chafe, Wallace. 1977. Accent and related phenomena in the Five Nations Iroquois languages. In L.M. Hyman, ed., Studies in Stress and Accent. Southern California Occasional Papers in Linguistics 4:169-181.

Hayes, Bruce. 1995. Metrical Stress Theory: Principles and Case Studies. University of Chicago Press.

Ladd, Robert. 1983. Phonological features of intonational peaks. Language 59:721-759.

Ladd, Robert. 1996. Intonational Phonology. Cambridge University Press.

Michelson, K. 1988. A Comparative Study of Lake-Iroquoian Accent. Dordrecht: Kluwer Academic Publishers.

Prince, Alan. 1983. Relating to the Grid. Linguistic Inquiry 14:19-100.

Woodbury, Hanni. 1992. Concerning the League: the Iroquois League Tradition as Dictated in Onondaga by John Arthur Gibson. Studies in Algonquian and Iroquoian Linguistics. Winnipeg, Manitoba.

Woodbury, Hanni. 2003. Onondaga - English / English - Onondaga Dictionary. University of Toronto Press.

Heike Lehnert-LeHouillier

602 Baldy Hall

Linguistics Department

University at Buffalo

Buffalo, NY 14260

hlehnert@buffalo.edu 